\title{
EDITORIAL
}

\section{Advancement in brain-machine interfaces for patients with tetraplegia: neurosurgical perspective}

\author{
Kejia Hu, MD,1,2 Firas Bounni, MD,1 and Ziv Williams, MD \\ 1'Department of Neurosurgery, Massachusetts General Hospital, Harvard Medical School, Boston, Massachusetts; and \\ 2Department of Microsurgery, Huashan Hospital, Fudan University, Shanghai, China
}

$\mathrm{T}$ ETRAPLEGIa results in the loss of motor function of all limbs and torso. It can affect basic motor function, sensation, respiration, digestion, bladder control, and other core autonomic functions. ${ }^{48}$ The most common cause of tetraplegia is spinal cord injury (SCI). It can also be caused by neuromuscular disorders such as amyotrophic lateral sclerosis (ALS) ${ }^{28,37}$ Although extensive efforts have been devoted to restoring damaged neural pathways following SCI, including physical rehabilitation, ${ }^{16,30}$ neuromodulation therapies, ${ }^{3}$ surgical procedures, ${ }^{4}$ and regenerative medicine, $, 29,35$ there currently remain few effective options for the restoration of motor or sensory function in individuals with tetraplegia.

Intracranial brain-machine interfaces (iBMIs), also known as intracranial brain-computer interfaces, are based on multidisciplinary approaches that combine neurophysiology, computer science, and bioengineering. Their principal aim is to restore motor and/or sensory capacities. Brain-machine interfaces use neural activity recorded from the brain to decode the motor intent of an individual in real time and, based on this, artificially produce the desired limb movement. ${ }^{33,42}$ Therefore, to obtain neuronal recordings, either through microelectrodes or electrocorticographic $(\mathrm{ECoG})$ grids, iBMI intrinsically requires neurosurgical access to functionally defined brain areas. ${ }^{5}$ Although most of these approaches have been tested in animal models, more recent studies have demonstrated the prospective use of iBMI technology in human patients with SCI. ${ }^{19}$ The rapid development and significant research efforts in this field suggest a more prominent role of iBMI in restorative neurosurgery.

\section{Motor Neuroprosthetics}

The field of iBMI development can be broadly divided into replacement and reanimation strategies. ${ }^{6}$ Both strategies decode neural signals recorded from the brain. For replacement, the aim is to control an artificial external actuator such as a robotic arm. For reanimation, the aim is to functionally bypass the disrupted neuronal pathways through direct functional electric stimulation (FES) of nerves and/or muscles of one's own paralyzed limb (Fig. 1).

For replacement approaches, high-performance upperlimb neuroprosthetic control achieved using an iBMI system has been demonstrated in patients with tetraplegia. ${ }^{5,21}$ Arguably the best clinical demonstration of this approach was recently given by a tetraplegic patient who was able to successfully control a robotic arm featuring 7 degrees of freedom to reach specific objects in space with high accuracy. ${ }^{13}$ Another participant in whom a 96-channel microelectrode array had been implanted in the motor cortex 5 years earlier further learned to control a robotic arm, enabling her to drink from a cup under her own volition. That study also demonstrated the feasibility of recreating useful prosthetic movements many years after injury. ${ }^{18}$

For reanimation approaches, prior work has shown that direct FES of nerves or muscles can be used to elicit movements of one's own paralyzed limbs. ${ }^{15,31}$ Therefore, by using BMI with custom-built high-resolution FES devices, the brain activity of a tetraplegic patient could be prospectively decoded and then used to control stimulation of a paralyzed limb. Here, the general idea would be to match an individual's motor intent (e.g., making an upward arm movement) based on decoded neuronal activity with FES of an appropriate muscle group or nerve that produces that same intended physical movement. Recently, such an approach has indeed been demonstrated in paralyzed patients, enabling them to play a simple guitar tune, ${ }^{8}$ or to perform self-paced movements such as drinking from a mug and self-feeding. ${ }^{1}$ Moreover, the prospective benefit of this restorative approach is the use of one's own limbs rather than an artificial or robotic one.

\section{Communication Neuroprosthetics}

In addition to the restoration of motor function, recent advancements in iBMI technology have raised the possibility of using iBMIs for communication. For example, pa-

ABBREVIATIONS ALS = amyotrophic lateral sclerosis; ECoG = electrocorticographic; FES = functional electric stimulation; iBMI = intracranial brain-machine interface; SCI 


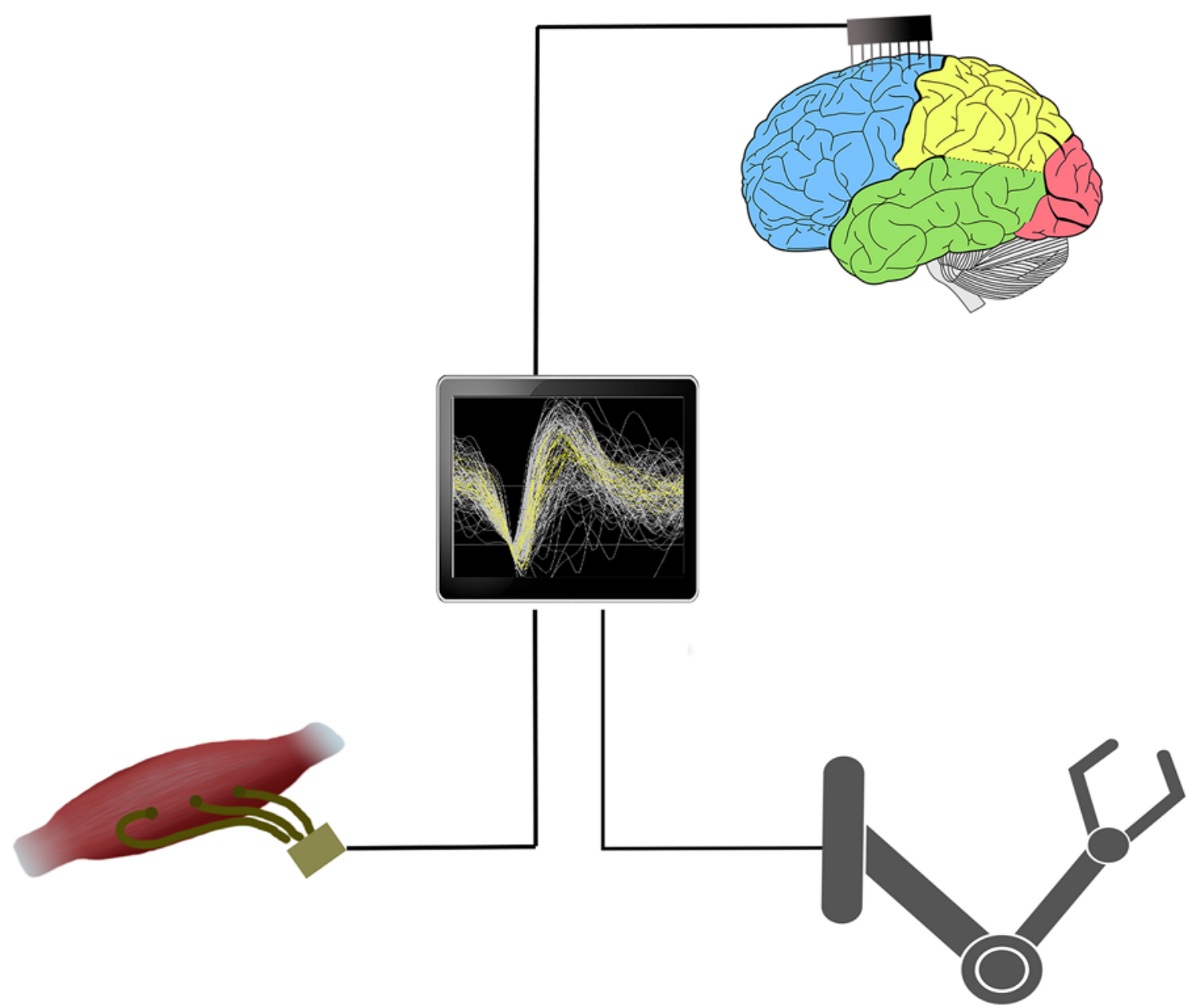

FIG. 1. Sketch of an iBMI system. Intracranial recordings of brain signals are used in cortical areas that are involved in control of movements. The activity of cortical neuronal ensembles is processed in real time, and then the outputs of these decoders are used to control the movements of a robot arm or the patient's own paralyzed muscle.

tients with ALS are often markedly limited by the inability to communicate through speech. So far, most technologies have focused on using preserved physical abilities to allow tetraplegic patients to communicate with the outside world. Examples include tracking eye movements to operate a mouse cursor or contracting one's cheek to spell words. These approaches, however, have a very low information bandwidth and are extraordinarily slow.

On the other hand, iBMIs often have access to hundreds of rapidly firing neurons and could, therefore, be potentially used for rapid communication. Currently, the most commonly used iBMIs for communication rely on translating brain activity into point-and-click control commands that allow one to sequentially select letters or words. ${ }^{17,22}$ With this technology, the highest speed and typing accuracy achieved by a patient with ALS was 2 letters per minute using subdural ECoG electrodes. ${ }^{47}$ The highest speed and accuracy achieved with a 96-channel microelectrode array was 39 correct characters, or approximately 8 words per minute. ${ }^{36}$

Another more recent approach has been aimed at recreating natural speech from brain activity. This represents a more direct way of communication and could potentially allow individuals to convey complex verbal messages in real time. Speech production and perception are processed differently in the frontal and temporal areas. ${ }^{12}$ Using this knowledge, prior ECoG-based studies have shown that speech articulation of the lips, tongue, jaw, and larynx can be decoded from the ventral premotor cortex. ${ }^{7}$ Other studies have also shown that additional representations, including word selection and phonetics formulation, could be reconstructed from auditory cortex, ${ }^{38}$ and that a complete set of English phonemes can be classified using highdensity arrays. ${ }^{32}$ Speech and language perception, however, are higher-order cognitive functions, and currently there is only a coarse understanding of how to decode speechrelated cortical activity. It is also not clear how to recreate other important aspects of speech communication such as accentuation and prosody. That being said, significant ongoing progress is being made in this field, and may provide an important supplement to current developments in motor-based iBMIs.

\section{Sensory Neuroprosthetics}

Sensory neural circuitry plays a critical role in executing motor tasks, and the lack of sensory feedback remains 
one of the largest limitations in most iBMI systems. ${ }^{5,27}$ Currently, most iBMIs rely solely on the patient's visual perceptions to provide feedback for motor control. Most natural movements are heavily guided by active somatosensation and proprioception, however, and these perceptions are often necessary for the effective, dynamic execution of voluntary movements. ${ }^{46}$ Accordingly, recent research has aimed to address this issue by providing artificial sensory feedback through direct cortical stimulation in real time. In these systems, mechanical sensors receive sensory information such as tactile, pressure, and robotic limb position. ${ }^{14}$ This sensory information is then coded into electrical impulses that activate focal areas within the somatosensory cortex of the participant. Therefore, with the delivery of sensory feedback through stimulation, individuals can adjust the hand aperture to move toward a targeted region or perceive the sensation of touch. In addition, nonhuman primate studies have shown that it is possible to combine intracortical long-term multielectrode recording and stimulation in the somatosensory cortex..$^{34,44}$ This approach will probably be necessary for effective neuroprosthetic control, and will require direct neurosurgical access to the somatosensory cortical circuitry.

\section{Future Perspectives}

The iBMI technology is rapidly growing, and is likely to become a major new component of restorative neurosurgery. However, the challenges that face iBMI research are still substantial. Current iBMI technology is generally cumbersome, requiring an in-house team of neurophysiologists and engineers, and is normally done within highly specialized laboratory settings. However, there is preliminary evidence to suggest that simpler epidural spinal cord stimulation may also be efficacious in producing electrophysiological improvements in patients with SCI. ${ }^{2,40}$ These devices are fully implantable and can be used, in principle, by patients at home. More recent studies in nonhuman primates have also addressed the feasibility of using implantable brain-controlled spinal stimulation systems to restore the function of one's own paralyzed limb, including restoration of voluntary grasp ${ }^{49}$ and $2 \mathrm{D}$ limb movements. ${ }^{41}$ This vision of a fully integrated wireless system would in turn make future iBMIs more practical. Other research has also demonstrated improved biocompatibility, ${ }^{23}$ and prevention of peri-electrode gliosis, ${ }^{43}$ which could improve the longterm viability of fully implantable iBMIs.

Most iBMI studies so far have principally focused on restoring upper-limb function, but similar approaches could also be used to restore lower-limb motor control. Restoring lower-limb motor functions such as walking and standing require control of leg and trunk muscles as well as precise maintenance of balance and reflex mechanisms to prevent falls..$^{20,39}$ These limitations currently remain the biggest challenge in iBMI development. The concept of brain-spinal interfaces for restoring ambulation, however, has been recently demonstrated in rhesus monkeys with unilateral SCI. Here, neuronal spiking activity was recorded from the leg area of the primary motor cortex, and decoded to ascertain the primate's intended leg movements. Then, electrical spinal stimulation was applied epidurally to produce extensions and flexions of the leg that matched the primate's intent. Without prior training, the primates were able to learn to volitionally control their paralyzed leg, and restored weight-bearing locomotion on a treadmill as early as 6 days postinjury. ${ }^{11}$ Although promising, further work is needed before this intervention can be tested in humans (i.e., who are bipedal and require precise, real-time balance control).

Finally, typical electrical stimulation systems have the general drawback of causing widespread neural activation, and the targeted muscle fibers are often prone to fatigue with repeating electrical stimulation. ${ }^{45}$ Optical stimulation is a different approach that may overcome the limitations of electrical stimulation. ${ }^{10,25,26}$ Using light energy, optical methods stimulate and record neural activity to provide artifact-free, noninvasive, and noncontact neurophysiological access. The addition of optogenetic techniques could also provide additional tissue target specificity. ${ }^{24}$ Taken together, these new emerging approaches point to an unprecedented level of prospective control over motor function, and may open the path toward practical clinical applications.

\section{Conclusions}

With the potential to restore limb movement, gait mobility, and somatosensation, iBMI systems offer patients with tetraplegia a prospective of near-immediate, long-term restoration of function. The benefit of these systems is that they are functionally specific, can be tailored in real time to optimize function, and can in principle functionally bypass any SCI irrespective of severity, location, or chronicity. Increased interdisciplinary collaboration among the many fields of science and engineering is of prime importance in advancing these prospective approaches from bench to bedside, with the eventual aim of restoring full sensorimotor functions in patients with tetraplegia.

https://thejns.org/doi/abs/10.3171/2017.5.FOCUS17244

\section{References}

1. Ajiboye AB, Willett FR, Young DR, Memberg WD, Murphy $\mathrm{BA}$, Miller JP, et al: Restoration of reaching and grasping movements through brain-controlled muscle stimulation in a person with tetraplegia: a proof-of-concept demonstration. Lancet [epub ahead of print], 2017

2. Angeli CA, Edgerton VR, Gerasimenko YP, Harkema SJ: Altering spinal cord excitability enables voluntary movements after chronic complete paralysis in humans. Brain 137:1394-1409, 2014

3. AuYong N, Lu DC: Neuromodulation of the lumbar spinal locomotor circuit. Neurosurg Clin N Am 25:15-23, 2014

4. Bednar MS: Tendon transfers for tetraplegia. Hand Clin 32:389-396, 2016

5. Bensmaia SJ, Miller LE: Restoring sensorimotor function through intracortical interfaces: progress and looming challenges. Nat Rev Neurosci 15:313-325, 2014

6. Borton D, Micera S, del R Millán J, Courtine G: Personalized neuroprosthetics. Sci Transl Med 5:210rv2, 2013

7. Bouchard KE, Mesgarani N, Johnson K, Chang EF: Functional organization of human sensorimotor cortex for speech articulation. Nature 495:327-332, 2013

8. Bouton CE, Shaikhouni A, Annetta NV, Bockbrader MA, Friedenberg DA, Nielson DM, et al: Restoring cortical control of functional movement in a human with quadriplegia. Nature 533:247-250, 2016 
9. Bryson JB, Machado CB, Lieberam I, Greensmith L: Restoring motor function using optogenetics and neural engraftment. Curr Opin Biotechnol 40:75-81, 2016

10. Canales A, Jia X, Froriep UP, Koppes RA, Tringides CM, Selvidge J, et al: Multifunctional fibers for simultaneous optical, electrical and chemical interrogation of neural circuits in vivo. Nat Biotechnol 33:277-284, 2015

11. Capogrosso M, Milekovic T, Borton D, Wagner F, Moraud EM, Mignardot JB, et al: A brain-spine interface alleviating gait deficits after spinal cord injury in primates. Nature 539:284-288, 2016

12. Cheung C, Hamiton LS, Johnson K, Chang EF: The auditory representation of speech sounds in human motor cortex. eLife 5:e12577, 2016

13. Collinger JL, Wodlinger B, Downey JE, Wang W, TylerKabara EC, Weber DJ, et al: High-performance neuroprosthetic control by an individual with tetraplegia. Lancet 381:557-564, 2013

14. Cronin JA, Wu J, Collins KL, Sarma D, Rao RP, Ojemann JG, et al: Task-specific somatosensory feedback via cortical stimulation in humans. IEEE Trans Haptics 9:515-522, 2016

15. Ethier C, Oby ER, Bauman MJ, Miller LE: Restoration of grasp following paralysis through brain-controlled stimulation of muscles. Nature 485:368-371, 2012

16. Fouad K, Tetzlaff W: Rehabilitative training and plasticity following spinal cord injury. Exp Neurol 235:91-99, 2012

17. Gilja V, Pandarinath C, Blabe CH, Nuyujukian P, Simeral JD, Sarma AA, et al: Clinical translation of a high-performance neural prosthesis. Nat Med 21:1142-1145, 2015

18. Hochberg LR, Bacher D, Jarosiewicz B, Masse NY, Simeral JD, Vogel J, et al: Reach and grasp by people with tetraplegia using a neurally controlled robotic arm. Nature 485:372375,2012

19. Hu K, Chen C, Meng Q, Williams Z, Xu W: Scientific profile of brain-computer interfaces: bibliometric analysis in a 10 year period. Neurosci Lett 635:61-66, 2016

20. Ijspeert AJ: Biorobotics: using robots to emulate and investigate agile locomotion. Science 346:196-203, 2014

21. Jackson A, Zimmermann JB: Neural interfaces for the brain and spinal cord-restoring motor function. Nat Rev Neurol 8:690-699, 2012

22. Jarosiewicz B, Sarma AA, Bacher D, Masse NY, Simeral JD, Sorice B, et al: Virtual typing by people with tetraplegia using a self-calibrating intracortical brain-computer interface. Sci Transl Med 7:313ra179, 2015

23. Jorfi M, Skousen JL, Weder C, Capadona JR: Progress towards biocompatible intracortical microelectrodes for neural interfacing applications. J Neural Eng 12:011001, 2015

24. Kravitz AV, Kreitzer AC: Optogenetic manipulation of neural circuitry in vivo. Curr Opin Neurobiol 21:433-439, 2011

25. Lee J, Ozden I, Song YK, Nurmikko AV: Transparent intracortical microprobe array for simultaneous spatiotemporal optical stimulation and multichannel electrical recording. Nat Methods 12:1157-1162, 2015

26. Llewellyn ME, Thompson KR, Deisseroth K, Delp SL: Orderly recruitment of motor units under optical control in vivo. Nat Med 16:1161-1165, 2010

27. Lobel DA, Lee KH: Brain machine interface and limb reanimation technologies: restoring function after spinal cord injury through development of a bypass system. Mayo Clin Proc 89:708-714, 2014

28. Loeffler JP, Picchiarelli G, Dupuis L, Gonzalez De Aguilar JL: The role of skeletal muscle in amyotrophic lateral sclerosis. Brain Pathol 26:227-236, 2016

29. Lu P, Wang Y, Graham L, McHale K, Gao M, Wu D, et al: Long-distance growth and connectivity of neural stem cells after severe spinal cord injury. Cell 150:1264-1273, 2012

30. Marsh BC, Astill SL, Utley A, Ichiyama RM: Movement re- habilitation after spinal cord injuries: emerging concepts and future directions. Brain Res Bull 84:327-336, 2011

31. Moritz CT, Perlmutter SI, Fetz EE: Direct control of paralysed muscles by cortical neurons. Nature 456:639-642, 2008

32. Mugler EM, Patton JL, Flint RD, Wright ZA, Schuele SU, Rosenow J, et al: Direct classification of all American English phonemes using signals from functional speech motor cortex. J Neural Eng 11:035015, 2014

33. Nicolas-Alonso LF, Gomez-Gil J: Brain computer interfaces, a review. Sensors (Basel) 12:1211-1279, 2012

34. O'Doherty JE, Lebedev MA, Ifft PJ, Zhuang KZ, Shokur $\mathrm{S}$, Bleuler H, et al: Active tactile exploration using a brainmachine-brain interface. Nature 479:228-231, 2011

35. Palma E, Reyes-Ruiz JM, Lopergolo D, Roseti C, Bertollini C, Ruffolo G, et al: Acetylcholine receptors from human muscle as pharmacological targets for ALS therapy. Proc Natl Acad Sci U S A 113:3060-3065, 2016

36. Pandarinath C, Nuyujukian P, Blabe CH, Sorice BL, Saab $\mathrm{J}$, Willett FR, et al: High performance communication by people with paralysis using an intracortical brain-computer interface. eLife 6:6, 2017

37. Pansarasa O, Rossi D, Berardinelli A, Cereda C: Amyotrophic lateral sclerosis and skeletal muscle: an update. Mol Neurobiol 49:984-990, 2014

38. Pasley BN, David SV, Mesgarani N, Flinker A, Shamma SA, Crone NE, et al: Reconstructing speech from human auditory cortex. PLoS Biol 10:e1001251, 2012

39. Pons JL, Moreno JC, Torricelli D, Taylor JS: Principles of human locomotion: a review. Conf Proc IEEE Eng Med Biol Soc 2013:6941-6944, 2013

40. Sayenko DG, Angeli C, Harkema SJ, Edgerton VR, Gerasimenko YP: Neuromodulation of evoked muscle potentials induced by epidural spinal-cord stimulation in paralyzed individuals. J Neurophysiol 111:1088-1099, 2014

41. Shanechi MM, Hu RC, Williams ZM: A cortical-spinal prosthesis for targeted limb movement in paralysed primate avatars. Nat Commun 5:3237, 2014

42. Shih JJ, Krusienski DJ, Wolpaw JR: Brain-computer interfaces in medicine. Mayo Clin Proc 87:268-279, 2012

43. Sridharan A, Rajan SD, Muthuswamy J: Long-term changes in the material properties of brain tissue at the implant-tissue interface. J Neural Eng 10:066001, 2013

44. Tabot GA, Dammann JF, Berg JA, Tenore FV, Boback JL, Vogelstein RJ, et al: Restoring the sense of touch with a prosthetic hand through a brain interface. Proc Natl Acad Sci U S A 110:18279-18284, 2013

45. Thrasher A, Graham GM, Popovic MR: Reducing muscle fatigue due to functional electrical stimulation using random modulation of stimulation parameters. Artif Organs 29:453-458, 2005

46. Tyler DJ: Neural interfaces for somatosensory feedback: bringing life to a prosthesis. Curr Opin Neurol 28:574-581, 2015

47. Vansteensel MJ, Pels EG, Bleichner MG, Branco MP, Denison T, Freudenburg ZV, et al: Fully implanted brain-computer interface in a locked-in patient with ALS. N Engl J Med 375:2060-2066, 2016

48. Weinshel SS, Maiman DJ, Baek P, Scales L: Neurologic recovery in quadriplegia following operative treatment. J Spinal Disord 3:244-249, 1990

49. Zimmermann JB, Jackson A: Closed-loop control of spinal cord stimulation to restore hand function after paralysis. Front Neurosci 8:87, 2014

\section{Disclosures}

The authors report no conflict of interest. 